\title{
Poly-Cross-Linked PEI Through Aromatically Conjugated Imine Linkages as a New Class of pH-Responsive Nucleic Acids Packing Cationic Polymers
}

\author{
Shun Chen and Tuo Jin* \\ Center for BioDelivery Sciences, School of Pharmacy, Shanghai Jiao Tong University, Shanghai, China
}

OPEN ACCESS

Edited by:

Aaron M. Mohs,

University of Nebraska Medical

Center, USA

Reviewed by:

Christopher D. Porada,

Wake Forest Institute for Regenerative

Medicine, USA

Hui Li,

University of Kentucky, USA

*Correspondence:

Tuo Jin

tjin@sjtu.edu.cn

Specialty section:

This article was submitted to Integrative and Regenerative

Pharmacology,

a section of the journal

Frontiers in Pharmacology

Received: 25 November 2015 Accepted: 15 January 2016

Published: 01 February 2016

Citation:

Chen S and Jin T (2016) Poly-Cross-Linked PEI Through Aromatically Conjugated Imine Linkages as a New Class of $\mathrm{pH}$-Responsive Nucleic Acids

Packing Cationic Polymers.

Front. Pharmacol. 7:15 doi: 10.3389/fphar.2016.00015
Cationic polyimines polymerized through aromatically conjugated bis-imine linkages and intra-molecular cross-linking were found to be a new class of effective transfection materials for their flexibility in structural optimization, responsiveness to intracellular environment, the ability to facilitate endosome escape and cytosol release of the nucleic acids, as well as self-metabolism. When three phthalaldehydes of different substitution positions were used to polymerize highly branched low-molecular weight polyethylenimine (PEI 1.8K), the product through ortho-phthalimines (named PPOP) showed significantly higher transfection activity than its two tere- and iso-analogs (named PPTP and PPIP). Physicochemical characterization confirmed the similarity of three polyimines in $\mathrm{pH}$-responded degradability, buffer capacity, as well as the size and Zeta potential of the polyplexes formed from the polymers. A mechanistic speculation may be that the ortho-positioned bis-imine linkage of PPOP may only lead to the straight trans-configuration due to steric hindrance, resulting in larger loops of intra-polymer cross-linking and more flexible backbone.

Keywords: steric structure, biodegraded polycationic carriers, imine, cross-link, gene delivery

\section{INTRODUCTION}

The critical hurdle for turning nucleic acids from therapeutic actives to practical medicines is the lack of therapeutically feasible delivery carriers (Kanasty et al., 2013; Yin et al., 2014). While the ability of viruses to deliver their genetic materials into host cells suggests that the chemical mechanism for designing synthetic carries of nucleic acids is existing in the nature, decades of research efforts including clinic trials of 20 candidates, have yet to reach a practically feasible system (Nguyen and Szoka, 2012; Ginn et al., 2013; Kanasty et al., 2013). To deliver RNA or DNA safely and efficiently into their site of action by systemic administration, a practically feasible synthetic carrier has to be fully functional, structurally simple, and easy to formulate. In fact, none of the systems examined in the clinical trials to date met these criteria. As an attempt to achieve this goal, we recently reported a self-assembled core-shell structured synthetic carrier system consisting a cationic polyplex core to accomplish the intracellular tasks of delivery and a tri-block copolymer membrane to achieve prolonged in vivo circulation and target cell recognition (Ge et al., 2015). We hypothesize that designing a core and a shell to address the intra- and intercellular tasks, 
respectively, will simplify the overall structure and ease the chemical assembly of a synthetic carrier of nucleic acids as compared with conjugating all the functional components to a polymer. This study is one of our continuing efforts in improving the polyplex core.

For intracellular delivery of genetic materials, the polyplex core needs to pack nucleic acids into a nanoparticle, facilitate endosomal escape, release of its cargos in cytosol (for siRNA), or in nucleus (for plasmids), and self-metabolize to non-toxic species (Overly et al., 1995; Pack et al., 2005; Duan et al., 2012). Our previously reported polyimine, polyspermine-4,5-imidazol imine (PSI), showed a highly efficient gene silencing probably due to the unique $\mathrm{pKa}$ of its imidazole ring (5.9) for which the nucleic acid-packing polymer degraded to non-toxic monomers in response to the endosomal pH ( 5.8) (Duan et al., 2012). For delivery of DNA plasmids, however, PSI was not as efficient as for siRNA because of its rapid self-degradation and nucleic acid release. For gene delivery, the polyplex forming cationic polymer may need a balanced $\mathrm{pH}$ responsiveness and $\mathrm{pH}$ resistance to delay the release of the nucleic acids for their approaching to the surface of nucleus. In the present study, therefore, we are trying to establish a spectrum between responsiveness and stability of the aromatic conjugated polyimines by varying the nitrogencontaining heterogeneous rings of different $\mathrm{pKa}$ and substitution positions which are involved in the polyimine linkages. Offering a broader choice along a responsiveness-stability spectrum versus a single point is the advance of this study over our previous report. Another objective of this study is, therefore, to examine whether internal cross-linking of the aromatically conjugated polyimines may be used to fine-tune this balance. Our hypothetic rationale is that certain levels of internal cross-linking may retard the overall dissociation the cationic polymer with the same $\mathrm{pH}$ responsiveness of the chemical bonds.

As an experimental approach to create different internal crosslinking density, phthalaldehydes of three different substitutions, tere-phthalaldehyde (TP), iso-phthalaldehyde (IP), and orthophthaldialdehyde (OP), were used as the linkers to polymerize branched low molecular weight (MW) polyethylene (PEI $1.8 \mathrm{KDa})$. Although phthalaldehydes are relatively toxic rather than non-toxic heterogeneous aromatic bis-aldehydes, such as imidazole formaldehydes, their three typical substitutions (tera-, iso- and ortho-) and stability provide defined steric differences. The resulted three polymers, poly-cross-linked PEI through tere-phthalimines, iso-phthalimines, and ortho-phthalimines (abbreviated to PPTP, PPIP, and PPOP, respectively) are same stoichiometrically but different sterically. Any differences in their physical/chemical properties and biological behavior should reflect the effect of the steric differences, and elucidating the steric effect may extend our ability in rational design of cationic polymer carriers of nucleic acids.

\section{MATERIALS AND METHODS}

\section{Materials}

Branched PEI 1.8 and $25 \mathrm{KDa}$ in average MW, were purchased from Sigma-Aldrich. TP, IP and OP were obtained from
TCI (Shanghai) Development Co., Ltd. Cellulose membranes for purifying polymeric products of desired MWs (MWCO $10,000 \mathrm{Da})$ were supplied by Thermo Scientific. All the anhydrous organic solvents were from Sigma-Aldrich, and all the reagents were used without further purification. Plasmid DNA (pDNA) encoding firefly luciferase pGL3-control (Promega) was amplified using EndoFree ${ }^{\mathrm{TM}}$ Plasmid Maxi (Qiagen). The sequences of luciferase pGL3-control siRNA were $5^{\prime}$-CUU ACG CUG AGU ACU UCG AdTdT-3' (sense strand) and 5'-UCG AAG UAC UCA GCG UAA GdTdT-3' (anti-sense strand).

\section{Synthesis and Characterization of the Polymers}

The designed polyimines were synthesized by condensation of PEI $1.8 \mathrm{~K}$ with the three linkers (TP, IP, and OP), respectively, as described by the reaction schemes in Figure 1 according to a reported method (Duan et al., 2012). Briefly, $2 \mathrm{mmol}$ of the linker molecules dissolved in $20 \mathrm{~mL}$ anhydrous ethylene dichloride was added dropwise into $1 \mathrm{mmol}$ PEI $1.8 \mathrm{~K}$ dissolved in $20 \mathrm{~mL}$ anhydrous ethylene dichloride under vigorous stirring at room temperature. After $24 \mathrm{~h}$ stirring, the solvent was removed by evaporation and the viscous residue was dissolved in deionized water and dialyzed through a cellulose membrane of the MW cutoff of 10,000 Da for additional 24 h. Finally, the polymers of differentiated MWs by dialysis were lyophilized for 2 days prior to storage at $-80^{\circ} \mathrm{C}$. Formation of the desired polyimines was confirmed using nuclear magnetic resonance (NMR), fourier transform infrared spectoscopy (FT-IR), and gel permeation chromatography (GPC) with polyethylene glycol (PEG) as the standard. The GPC of the polymers were recorded on an Agilent 1260 HPLC system equipped with a refractive index detector (RID) and a thermostatic gel permeation chromatography column PL aquagel-OH. The column temperature was maintained at $40^{\circ} \mathrm{C}$ and the mobile phase was $\mathrm{ddH}_{2} \mathrm{O}$ at the flow rate of $1 \mathrm{~mL} / \mathrm{min}$. Nitrogen

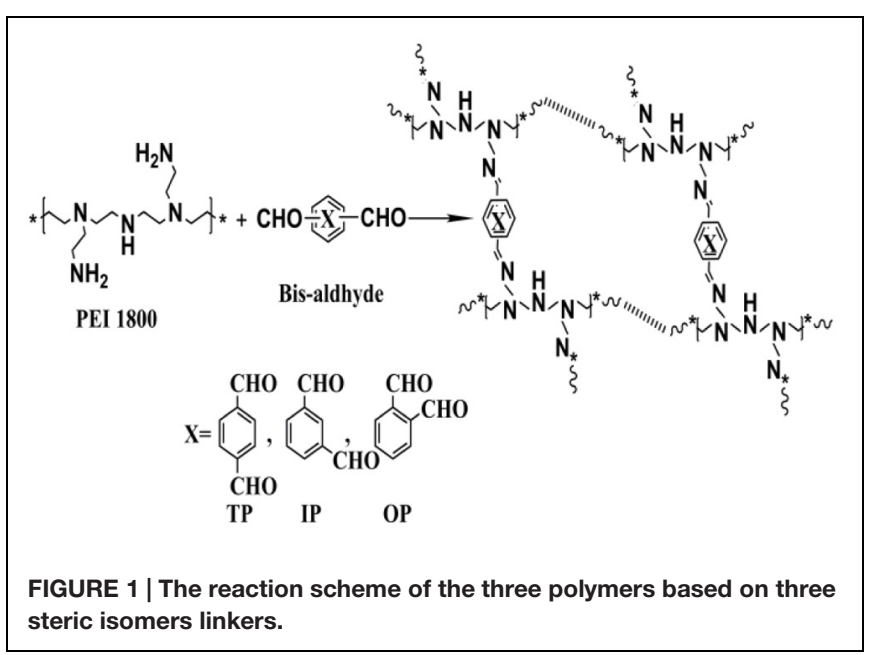


content was measured by Elemental Analyzer (Vario-EL Cube, Elementar).

\section{Degradation and Buffering Capacity of the Polymers}

Each of the cationic polyimines was added in deionized water to reach a concentration of $2 \mathrm{mg} / \mathrm{mL}$. Then, the solution was divided to four parts, and $\mathrm{pH}$ of the four was adjusted to 5.0, 6.0, 7.4, respectively, by titration with $\mathrm{HCl}$. After the solutions were incubated at $37^{\circ} \mathrm{C}$ for 3 days, MW changes of the polymers were measured by GPC. The buffering capacity of the polyimines was determined by titrating the polymer solutions from $\mathrm{pH} 9.0$ to $\mathrm{pH}$ 4.0. Briefly, $0.1 \mathrm{mg} / \mathrm{mL}$ aqueous solution of each of the polyimines were prepared, and titrated with $0.1 \mathrm{M} \mathrm{HCl}$ at $25^{\circ} \mathrm{C}$. The $\mathrm{pH}$ values were recorded with a $\mathrm{pH}$ Meter (Mettler Toledo). The buffering capacity was defined as the percentage of amino groups becoming protonated from 7.4 to 5.0 (mimic the $\mathrm{pH}$ change from the extracellular environment to the lower $\mathrm{pH}$ of the endosome-lysosome system; Overly et al., 1995; Convertine et al., 2010).

\section{Cytotoxicity of the Polyimines}

The cytotoxicity of the polyimines was assessed by cell viability using 3-(4,5-dimethyl-2-thiazolyl)-2,5-diphenyl-2H-tetrazolium bromide (MTT) assay and hemolytic activity measurement. For MTT assay, each of the three cell lines, COS-7, HeLa and SMMC7721, was incubated with the polymers solutions (dissolved in PBS) of gradually increasing concentrations from 10 to $100 \mu \mathrm{g} / \mathrm{mL}$ for $4 \mathrm{~h}$. Then, $20 \mu \mathrm{L}$ of MTT $(5 \mathrm{mg} / \mathrm{mL})$ solution was added into each well and incubated at $37^{\circ} \mathrm{C}$ for additional $6 \mathrm{~h}$. Prior to absorbance reading, the medium of each well was replaced with $150 \mu \mathrm{L}$ of DMSO and the plate was shaking for $10 \mathrm{~min}$ at room temperature. Absorbance at $570 \mathrm{~nm}$ was measured with Microplate Reader (3M, USA), and reference at $630 \mathrm{~nm}$. The MTT value of untreated cells was considered as $100 \%$ cell viability. All transfection and toxicity assays were performed in triplicate. Three linker molecules were investigated at the same process, concentrations from 1 to $20 \mu \mathrm{g} / \mathrm{mL}$ with the same process. Hemolytic activity was utilized to determine membrane lysis of mice erythrocytes by the three polyimines. According to previously reported method, nine $\mathrm{BALB} / \mathrm{c}$ female mice (5-week-old, weight $20 \pm 2 \mathrm{~g}$, obtained from the Institute of Zoology, China Academy of Sciences) were used as the model. The mice were cared for under Specific pathogen Free (SPF) environment in the laboratory animal facility in the school of pharmacy, Shanghai Jiao Tong University. The animal experiments were conducted according to the guidelines approved by the Regulations for the Administration of Affair Concerning Laboratory Animals and in adherence to the National Regulation for Care and Use of Laboratory Animals of China. Mice erythrocytes were separated from fresh citrate-treated blood and washed in PBS by four centrifugation cycles, each at $2000 \mathrm{rpm}$ for $10 \mathrm{~min}$. The red blood cells (RBCs) were diluted in PBS to a final concentration of $2 \times 10^{8}$ erythrocytes $/ \mathrm{mL}$. Polymer solutions of different concentrations in PBS $(80 \mu \mathrm{L})$ and the same volume
$(80 \mu \mathrm{L})$ of $\mathrm{RBC}$ suspension were added consequently in to each well of a 96-well plate, followed by incubation at $37^{\circ} \mathrm{C}$ for 45 min under constant shaking. The cells were also treated with PBS and 1\% Triton X-100 as negative and positive control. After centrifugation at $2000 \mathrm{rpm}$ for $10 \mathrm{~min}$, the supernatant in each well was collected and analyzed for the content of hemoglobin released from the erythrocytes using a Microplate Reader (SpectraMax M3, USA) at the wavelength of $450 \mathrm{~nm}$. The experiment was repeated three times for calculation of the polymers relative to PBS and Triton X-100.

\section{Preparation and Characterization of the Polyplexes}

Polyplexes were prepared by mixing the polymer solution into a pDNA solution at pre-set nitrogen-to-phosphor $(\mathrm{N} / \mathrm{P})$ ratios. Particle size and Zeta potential was determined using dynamic light scattering (DLS) particle sizer (Brookhaven Instruments Corporation 90 Plus). The morphology of polyplexes was imaged under a transmission electron microscope (TEM). Gel electrophoresis, another assay to confirm polyplex formation, was carried out on a $1 \%(\mathrm{w} / \mathrm{v})$ agarose gel pretreated with $0.5 \mathrm{mg} / \mathrm{mL}$ ethidium bromide in $1 \times$ Tris-boric acid-EDTA (TBE) buffer at $110 \mathrm{~V}$, and result was imaged using a UV illuminator (Tanon 2500 Gel Image System).

\section{Transfection In Vitro}

Transfection experiments were performed on three cell lines, COS-7, HeLa, and SMMC7721, using plasmid pGL3 luciferase as the reporter gene. Cells were cultured to $90 \%$ confluence in Dulbecco's Modified Eagle's Medium (DMEM, Invitrogen Co., USA) containing 10\% FBS (Invitrogen Co., USA) and $1 \%$ penicillin/streptomycin (Stock 10,000 U/mL, 10,000 ug/mL, Invitrogen Co., USA). Cells were seeded into 48-well plates at a density of $1 \times 10^{5}$ cells/well $24 \mathrm{~h}$ before transfection. When the cells were approximately $80 \%$ confluence, washed the cells by PBS twice, and added $250 \mu \mathrm{L}$ medium without serum at each well. $50 \mu \mathrm{L}$ polyplexes with different polymer/DNA $\mathrm{N} / \mathrm{P}$ ratios ranging from 10:1 to 250:1 were gently overlaid into the wells. Each well contains $500 \mathrm{ng}$ pDNA. The plates were incubated at $37^{\circ} \mathrm{C}$ in a $5 \% \quad \mathrm{CO}_{2}$ incubator for 4 hours. After incubation, the transfection medium was replaced with $0.5 \mathrm{~mL}$ fresh complete medium. The plates were incubated for $48 \mathrm{~h}$ under the same conditions as previously. Expression of luciferase was measured according to the instruction. The cells were washed twice with PBS and lysed with lysis buffer $(1 \times$, Promega). Cell debris was removed by centrifugation at 12,000 rpm for 3 min (Eppendorf 5810R Centrifuge, Germany) and $20 \mu \mathrm{L}$ of the supernatant add $20 \mu \mathrm{L}$ substrate solution (Luciferase Assay System, Promega). The luminescence was measured by Single Tube Luminometer (Berthold Detection Systems $\mathrm{GmbH}$ ). The total protein concentrations in cell lysates were determined using Micro BCATM Protein Assay Kit (Thermo Scientific Pierce). Luciferase activity was assessed by relative light units (RLUs) per protein concentrations (mg). 


\section{Statistical Analysis}

The quantitative data obtained in the experiments of this study were analyzed using the Student $t$-test. For comparative figures, significant differences at the level of $* p<0.05$ and very significant differences at the level of $*^{*} p<0.01$.

\section{RESULTS}

\section{Synthesis and Confirmation of Polyimines}

The synthesis of the polyimines was a one-step polymerization as described in Figure 1. Formation of three polymers were confirmed by their ${ }^{1} \mathrm{H}-\mathrm{NMR}$ spectra shown in Figure $\mathbf{2 A}$, wherein the chemical shift of the proton over $\delta 10.0$ assigned for aldehyde groups disappeared and those at $\delta 7.45-\delta 7.70$ for imine groups appeared instead, indicating the condensation of the aldehydes and primary amines. The formation of the imine linkages were also confirmed by the absorbance at $\sim 1640 \mathrm{~cm}^{-1}$ in the FT-IR spectra for all the three polymers (Figure 2B). The average MW of PPTP, PPIP and PPOP were determined by GPC to be $21.5,20.5$, and $21.1 \mathrm{KDa}$, respectively (Figure 3A).

The results of the nitrogen contents were determined by elemental analyzer and shown in Table 1. For all the three polymers, nitrogen contents of reduced similarly by one-third. The decrease of nitrogen contents was resulted from the
A

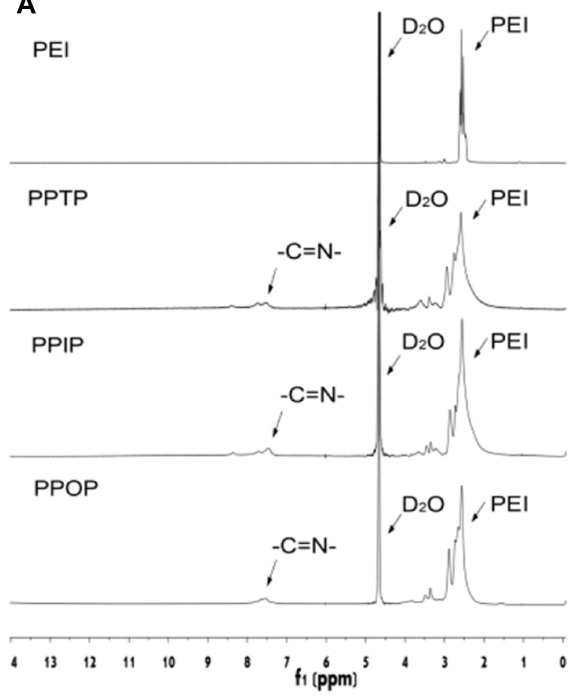

B
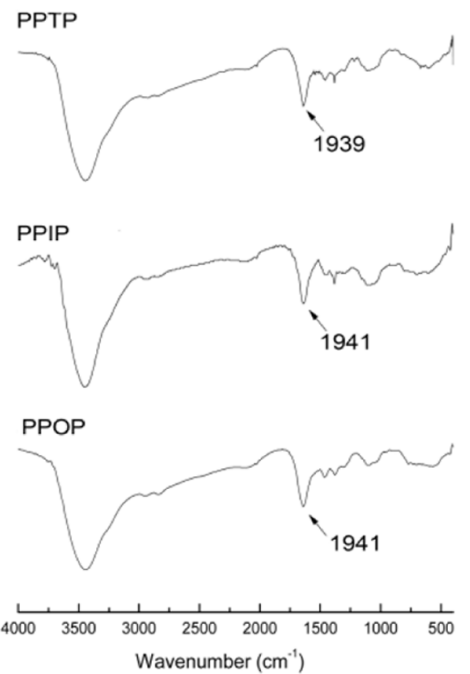

FIGURE 2 | Structure characterization of the three polymers. (A) ${ }^{1} \mathrm{H}-\mathrm{NMR}$ and (B) fourier transform infrared spectroscopy (FT-IR).

A

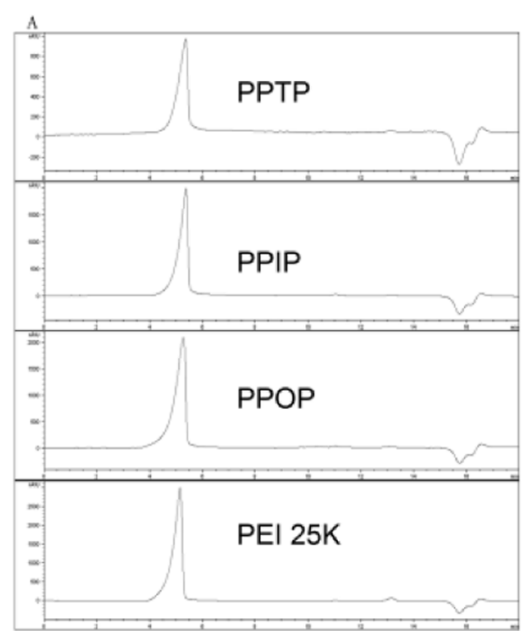

B

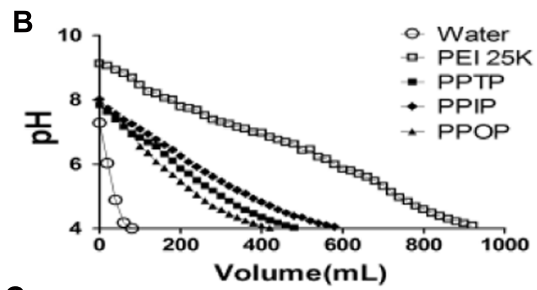

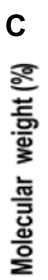

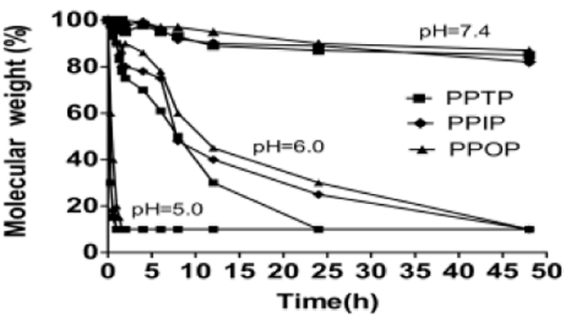

FIGURE 3 | (A) Molecular weight (MW) was calculated by GPC methods. (B) pH buffering capacity of the polyethylenimine (PEI) derivate from pH 9 to 4 with the same concentration $(0.1 \mathrm{mg} / \mathrm{ml})$ and $(\mathbf{C})$ Degradation of the three polymers in different $\mathrm{pH}$ conditions. 
TABLE 1 | Nitrogen contents of different cationic polymers.

\begin{tabular}{lc}
\hline Polymer & N (\%) \\
\hline Polyethylenimine (PEI 25K) & 31.3 \\
PEI 1.8K & 31.9 \\
Tere-phthalimines (PPTP) & 22.2 \\
Iso-phthalimines (PPIP) & 23.1 \\
Ortho-phthalimines (PPOP) & 22.3 \\
\hline
\end{tabular}

conjugation of the phthaladehyde linkers that contributed mass without nitrogen. Even if the nitrogen content dropping may not be accurate enough quantitatively, the similarity between the three polymers is consistent with their similar MW determined by GPC.

\section{Buffering Capability and $\mathrm{pH}-$ Responsive Degradation of Polyimines}

The buffering capability of the PEI $25 \mathrm{~K}$ and the three polyimines were determined by acid-base titration. As shown in Figure 3B, for the same $\mathrm{pH}$ drop, all the three polyimines required less acid than PEI $25 \mathrm{~K}$ of identical w/v concentration. This titration result is consistent with that of nitrogen content measurement (Table 1), by reacting with phthaldialdehydes, the amino content of PEI $1.8 \mathrm{~K}$ per unit mass dropped significantly as the drop in the amount of titrating acids for the same $\mathrm{pH}$ declining. Between the polyimines of similar structure and amino content, however, the difference in buffer capacity was less significant, although that of PPTP and PPIP seemed to be slightly larger than PPOP.
In addition to buffer capacity, the three polyimines showed similar degradation rate at given $\mathrm{pH}$ (Figure 3C). Dropping of their average $\mathrm{MW}$ was accelerated by the decrease in $\mathrm{pH}$. At $\mathrm{pH} 7.4$, the change in average of $\mathrm{MW}$ of the polyimines was negligible, but at $\mathrm{pH} 6.0$, similar to that of endosomes, the MW dropped to $50 \%$ of its original in $15 \mathrm{~h}$. Further reducing $\mathrm{pH}$ to 5.0 resulted in shortened time duration for the MW to reach the $50 \%$ of the original from 15 to 0.5 hours (Figure $3 \mathrm{C}$ ).

Compared with our previously reported polyimine, PSI (Duan et al., 2012), the three polyimines in the present study degraded in the same rate at $\mathrm{pH} 6.0$ and 5.0, but much more stable at $\mathrm{pH} 7.4$ (for PSI, the time to reach 50\% MW dropping was 48 hours). We also examined the degradability of several polyspermine imines polymerized through aromatically conjugated bis-imine linkages of various $\mathrm{pKa}$, and found no significant differences in the rate of $\mathrm{pH}$-responsive degradation between them (Unpublished data.). These results lead us to suggest that intramolecular crosslinking is an effective way to retard the overall dissociation of the overall backbone of polyimines polymerized through aromatically conjugated imine linkages.

\section{Cytotoxicity of the Polyimines}

The cytotoxicity of the three polyimines was examined by the viability of COS7, HeLa, SMMC-7721 cells treated with the polymers of various concentrations. As indicated in Figure 4A, although all the three polyimines were less toxic than PEI 25K as expected, PPOP showed significantly higher cytotoxicity than the other two. While some differences appeared between cell lines, the viability of all the cells
A
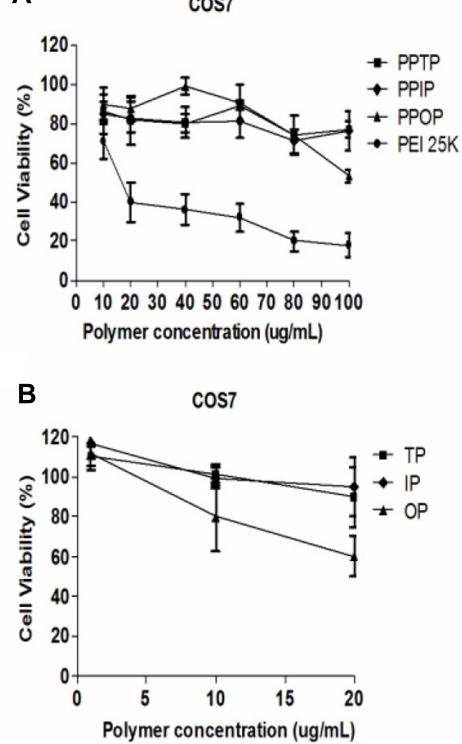

HeLa
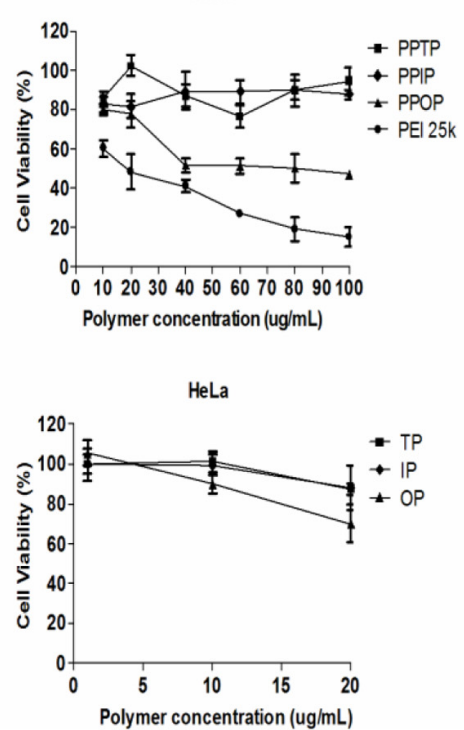

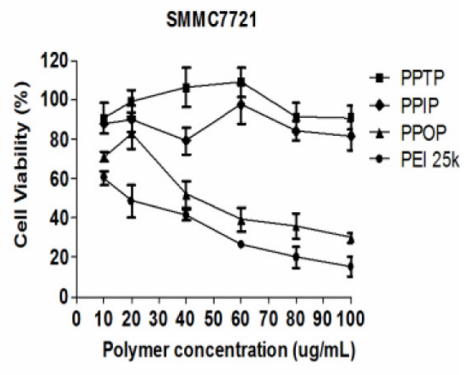

SMMC7721

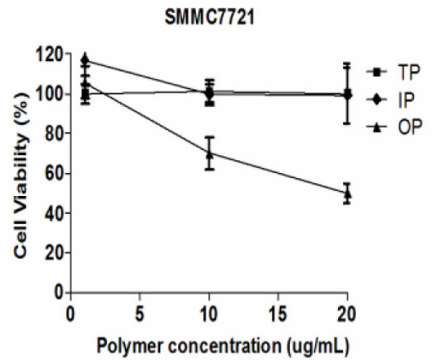

FIGURE 4 | (A) Cell viability of three polymers in three cell lines with different concentrations. (B) Cell viability of three linker molecules in three cell lines with different concentrations. Considered that nitrogen contents change between PEI 1.8K and three polymers, the linker molecules contents could be about $20 \%$ of the polymers after calculation. The concentrations included the degraded linkers concentrations supposed that three polymers from (A) had degraded completely. 
dropped significantly when the concentration of PPOP was increased, especially for HeLa and SMMC7721 cells (Figure 4A). This polymer concentration-depended viability change was not observed for those cells treated with PPTP and PPIP (Figure 4A). To elucidate the cause for the severer cytotoxicity of PPOP, the cells were treated with the three difference precursors, TP), IP, and OP of corresponding concentrations, followed by MTT assay for cell viability. The result (Figure 4B) indicated that only OP caused cell viability drop among the three phthalaldehydes. However, the orders of the cells in viability changes resulted from the polymers and the phthalaldehydes were not consistent with each other (Figure 4). For the treatment by the polymeric PPOP, the viability of COS7 cells remained almost unchanged, but severe viability drop were observed for HeLa and SMMC772 cells. For the treatment by the OP monomer, quite differently, COS7, HeLa, and SMMC7721 cells showed a significant viability drop. Although the OP monomer showed some levels of cytotoxicity, it seems that the decreased cell viability by polymeric PPOP cannot be attributed to the effect of OP.

To further investigate the mechanism of cytotoxicity of the polymers, hemolytic activity of these cationic polymers was measured. Lyse RBCs in vitro was also used as an indicator of endosomelytic property (Plank et al., 1994). Cationic polymers above certain concentration may induce hemolytic rupture of red blood cells (RBC) and release of hemoglobin by adsorbing on the anionic cell surface(Rekha and Sharma, 2009). Density of the positive charges and flexibility of the polymer backbone to enable the charged groups to approach their adsorption sites (the negative charge) on the cell surface determine the release rate of hemoglobin (Hong et al., 2006; Lv et al., 2006). As shown in Figure 5, when the three polyimines and PEI $25 \mathrm{~K}$ were added to culture of RBC, with Triton-X100 and PBS buffer as the references, the cells treated with PPOP and PEI $25 \mathrm{~K}$ showed higher hemoglobin release than those treated with PPTP and PPIP as the polymer concentration increased. In fact, the hemolytic activity of PPTP and PPIP appeared to be same as PBS, indicating negligible damage of the RBC membrane. Assuming the electrostatic interaction between a positively charged polymer and the negatively charged cell membrane causes lytic rupture of the cell, we may conclude at this stage that PPOP of polymeric state was more responsible for the cytotoxicity shown in Figure 4. If this argument is true, the fact that the structurally similar polyimines showed different hemolytic activity indicates that steric structure determined the cytotoxicity.

\section{Polyplexes Formation from Polyimines and DNA}

The formation of polyplexes by mixing DNA with each of the three synthesized polyimines was confirmed using electrophoresis, dynamic light scattering (DLS), and TEM. As a typical method, agarose gel electrophoresis was performed to assay the polyplex formation as well as the DNA condensing
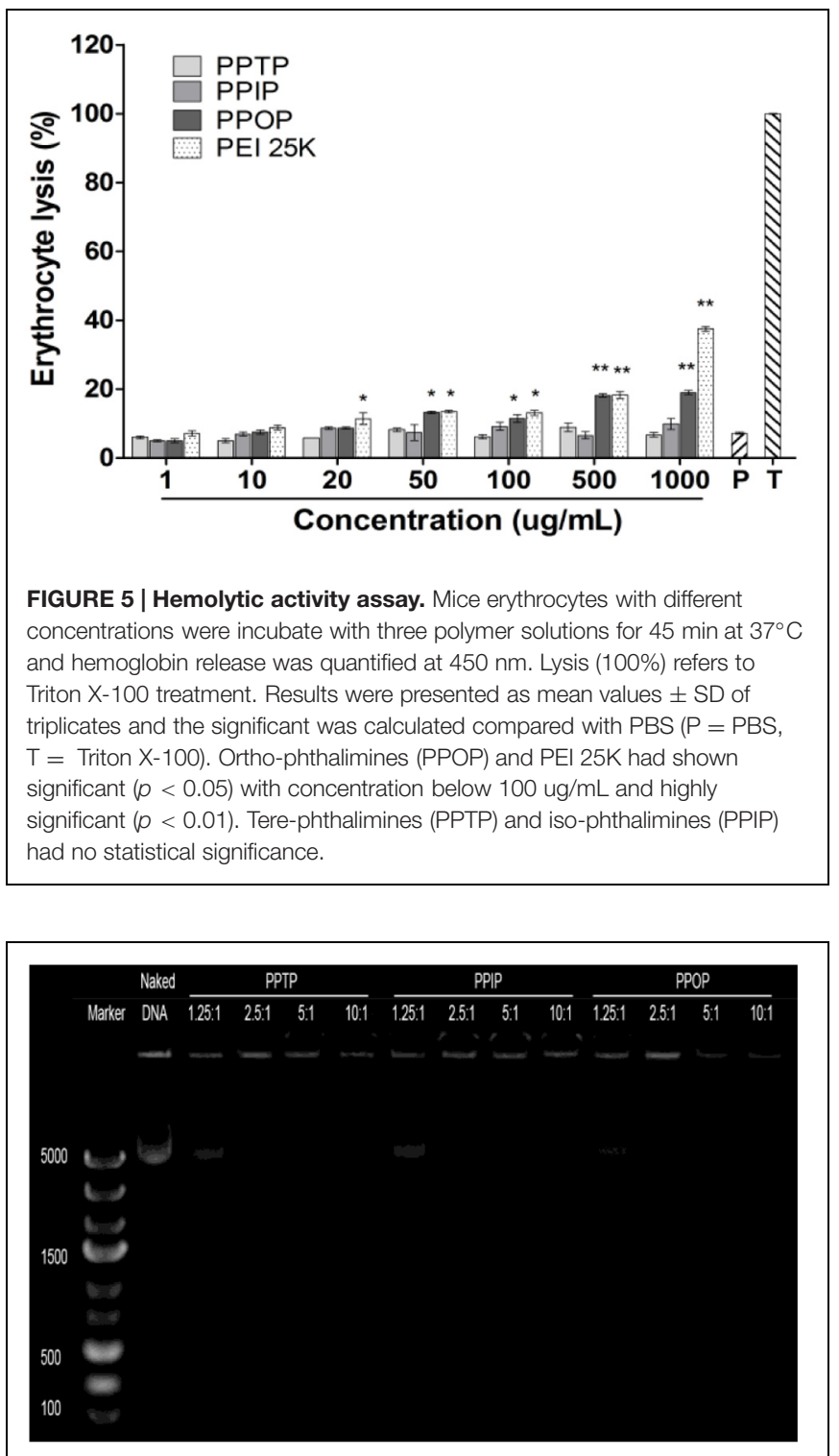

FIGURE 6 | DNA-binding assay. The DNA binding abilities of the polymers were examined by agarose electrophoresis with different N/P ratio with DNA.

capability, the minimal N/P ratio for complete capture of nucleic acids. As shown in Figure 6, the electrophoresis band for the tested plasmid DNA became completely invisible when the N/P ratio reached 2.5 for all the three polyimines. This result suggests that under the condition and concentration of the electrophoresis experiment, the three cationic polymers showed the similar DNA packing capability.

The DLS measurement indicated, on the other hand, that the polyplexes formed from the three polyimines were $120 \sim 150 \mathrm{~nm}$ in diameters for all the N/P ratios (Figure 7A), and -5 to $0 \mathrm{mV}$ to $15-20 \mathrm{mV}$ in Zeta potential for the polymer/DNA (N/P) ratio was increased from 2.5:1 to 50:1 (Figure 7B). The increasing Zeta potential and the invariable diameter of the polyplexes up to the $\mathrm{N} / \mathrm{P}$ ratio of 50:1 are consistent with the well observed dynamic 

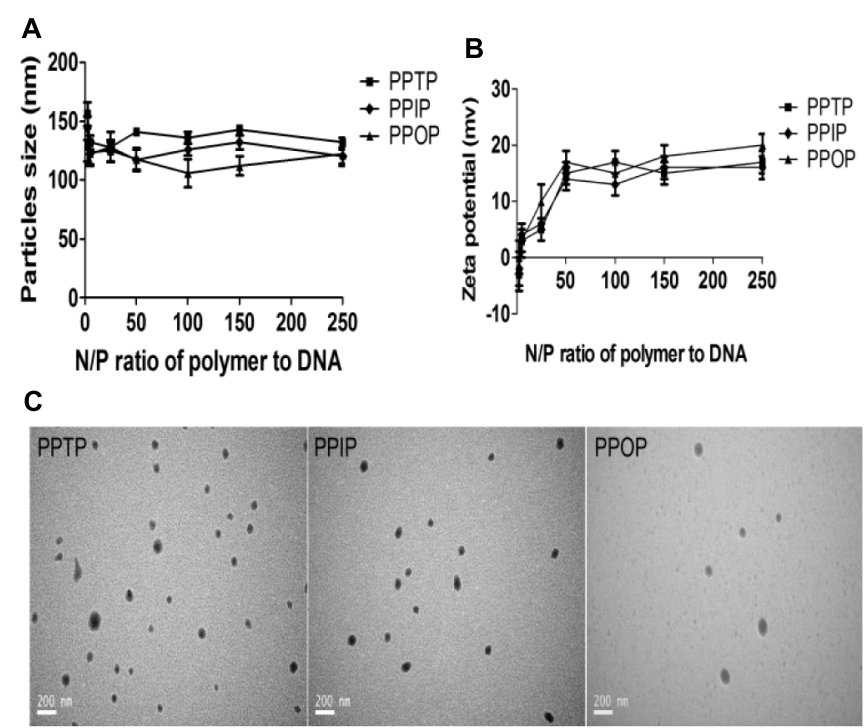

FIGURE 7 | Physiochemical properties of the polyplexes prepared with the three polymers and plasmid DNAs. (A) Particles size, (B) Zeta potential, and (C) transmission electron microscope (TEM).

complexation process that adsorption of cationic polymer result in elevation of Zeta potential, increased in polymer repulsion, and reduce in polyplex mass (Mintzer and Simanek, 2009). Continuous increase in the N/P ratio from 50:1 to $250: 1$ did not result in further elevation of Zeta potential, suggesting that additional cationic polymer did not involve in the polyplex formation under the polymer concentration of DLS measurement (Figures 7A,B). As expected, the polyplex size measure using TEM in dry state was smaller that that determined by DLS in aqueous suspension (Figure 7C). The larger size of cationic polyplexes in aqueous suspension may be attributed to the repulsion between the excess of cationic polymer, which consists with our earlier report that cationic polyplexes have plenty of room to shrink in case the position charges are neutralized by oppositely charged surface functionalization components (Ge et al., 2015).

\section{In Vitro Transfection Activity}

The three cationic polyimines were used to pack and transmit luciferase plasmid pGL3 as the reporter gene to each of the cell lines, COS-7, Hela and SMMC7721, followed by determination of luciferase activity. As shown in Figure 8, the maximum transfection efficiency for PPOP achieved at w/w ratio of 5:1 (N/P ratio of 40:1) was comparable to that of PEI $25 \mathrm{~K}$ in all the three cell lines. For PPTP and PPIP, the transfection efficiency was more than one degree of magnitude lower than PPOP and PEI 25K. Although for SMMC7721 cells, PPTP and PPIP reached the similar level transfection, the $\mathrm{w} / \mathrm{w}$ ratio was as high as 20:1 (N/P ratio of 160:1). Since the three polyimines possess the same MW, buffer capacity, amino group content, and $\mathrm{pH}$-responded degradation rate, the superior transfection activity of PPOP over PPTP and PPIP could only be resulted from their steric differences in structure. We hypothesize that steric hindrance of the ortho-substituted bis-imine linkages in PPOP took only the trans-configuration, which lead to large loops of intra-molecular cross-linking as compared with PPTP and PPIP. And the larger loops allow the overall backbone of the polyimine to be more flexible and accessible to nucleic acids. More discussion will be given in the Section "Discussion".

Similarly as many cationic polymers, when further increasing the polymer concentration above the optimum polymer to nucleic acid ratio $(\mathrm{w} / \mathrm{w}=5: 1)$ resulted in activity declining for PPOP (Figure 8), suggesting increased cytotoxicity. It is often observed that gene transfection by cationic polymers is facilitated by an optimum polymer-to-DNA ratio to ensure sufficient DNA packaging and tolerable cytotoxicity. Among the three structurally similar polyimines, however, the optimum polymer-to-DNA ratios were considerably different between the structurally similar PPOP and the other two. Both of the transfection assay and cell viability assay support the speculation that PPOP possesses more flexible overall backbone to facilitate the interaction with the anionic charges of DNA as well as cell membranes.

\section{DISCUSSION}

While degradation in response to cellular environment is recognized to be a highly favored criterion in designing cationic polymers for packing nucleic acids, materials in this category are limited to few classes such as poly- $\beta$-amino acid esters and polymers linked with di-sulfur bonds (Akinc et al., 2003; Lee and Kim, 2014). Majority of research efforts have been focusing to conjugate functional components for inter-cellular trafficking to cationic polymers. However, multi-steps involved covalent conjugation of functional groups onto a cationic polymer greatly limits the flexibility in designing the nucleic acid 


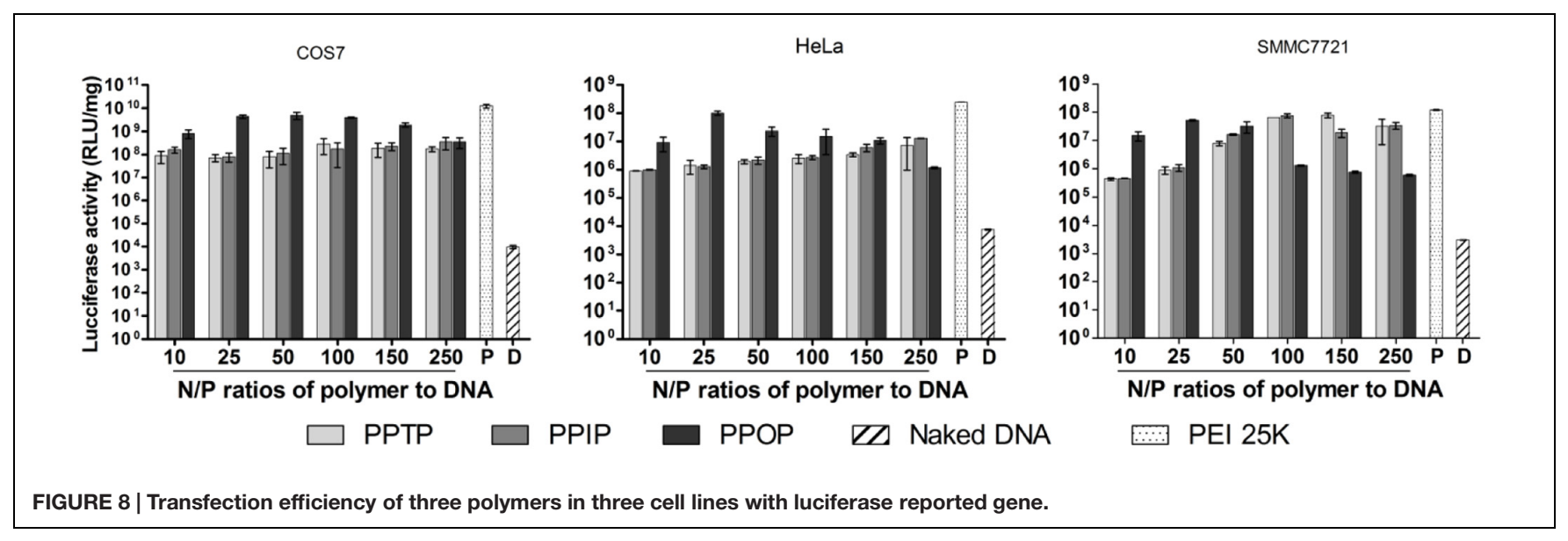

packing polymer itself. Our recently invented self-assembly of a tri-block copolymer membrane around each cationic polyplex of any content has provided a very convenient method to protect and functionalize polyplexes at post-polyplexing stage (Ge et al., 2015). Covalent conjugation of inter-cellular delivery components is, therefore, no longer necessary, and design of cationic polymers can be focused on the intracellular tasks only.

By getting rid of the multi-step reactions to link cell targeting components, generally instable cationic polyimines become feasible as $\mathrm{pH}$-responsive nucleic acid packing materials. When conjugated with an aromatic ring, certain levels of acid-resistance of the imine linkages of the polymers may be achieved (Duan et al., 2012). It has been reported that silencing a gene by siRNA or expressing a gene by plasmids demand different intra-cellular functions from a carrier system (Pack et al., 2005). The former is benefited by rapid release with sufficient number of copies of siRNA from the carrier into the cytoplasm of target cells, while the latter requires sustained stability of the polyplexes to facilitate intra-cellular movement approaching the nucleus surface (Lukacs et al., 2000; Lee et al., 2009; Stalder et al., 2013). Endosomes transport cargoes from extracellular environment to the degradative lysosome. It take distinguished time for early endosomes mature to form late endosomes with increasingly acidic mainly through the activity of the V-ATPase (Lafourcade et al., 2008; Stenmark, 2009). For nucleic acids, endosome releasing was very important before endosome-lysosome fusion. Therefore, balancing between the $\mathrm{pH}$-responsiveness and $\mathrm{pH}$ resistance according to the delivery purpose become a factor in designing nucleic acid packing polyimines.

For a $\mathrm{pH}$-responsive polyimine, there may be two ways to improve its stability, forming a aromatically conjugated imine linkage which has relative low $\mathrm{pKa}$ in terms of proton adsorption, and forming some internal cross-linking to retard overall dissociation of the polymer backbone. In the present study, the second strategy was utilized and highly branched small molecular PEI (PEI 1.8K) was used as the amino group-bearing building block to form the cross-linking loops. At this stage, we recognized that substitution position of the aromatic bis-imine linkage played a critical role in determining gene transfection activity of the polyimine carriers. Since the three poly-phthalimines are the same in chemical bonding, average $\mathrm{MW}$, as well as $\mathrm{pH}$ depended degradation rate (Figures 2 and 3), the significant differences in gene transfection efficiency between the orthopositioned PPOP and the other two may only be attributed to the cross-linking configurations. We therefore hypothesize that the ortho-positioned phthalimine structure can only be formed in the straight trans-configuration due to steric hindrance, resulting in larger cross-linking loops within PPOP (Figure 9). For the bisimine linkage in PPTP and PPIP, the steric availability of both the trans- and cis-configurations allowed small cross-linking loops to be formed, resulting in firmer overall backbones (Figure 9). Since packing RNA or DNA into nano-particulate polyplex is achieved through electrostatic complexation between cationic polymers and anionic nucleic acids, flexibility of the polymer backbone represents better accessibility of the oppositely charged species to each other and more efficient packaging.

For a cationic carrier polymer of a given structure, its gene transfection activity is often associated with its cytotoxicity, because the mechanisms for the polymer to encapsulate anionic nucleic acids and to adsorb on negatively charged cell membranes

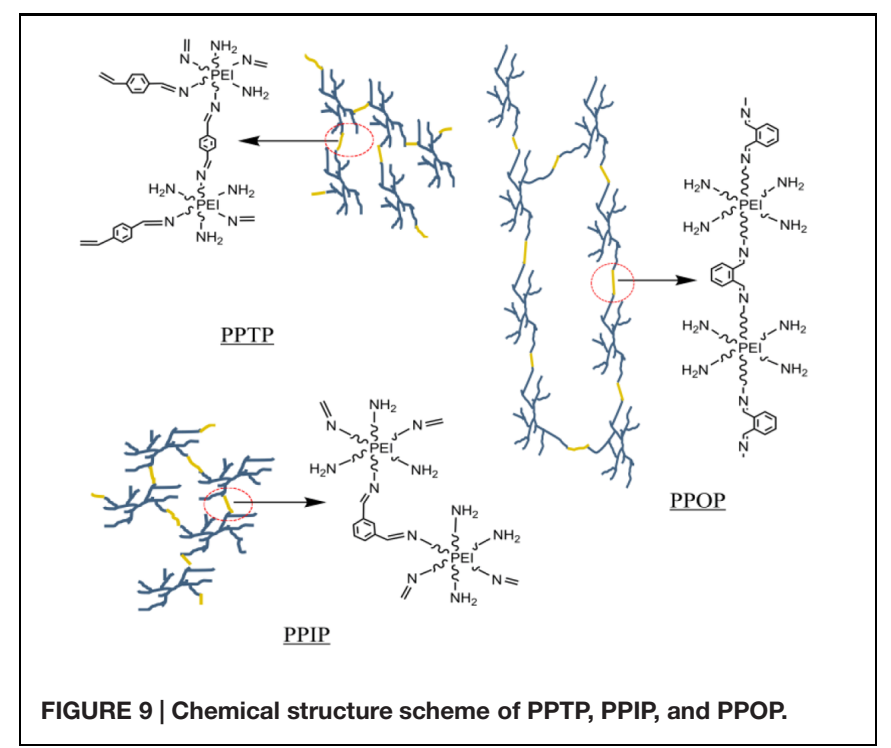


are the same, electrostatic adhesion. This mechanism applied to the three polyethylene phthalimines of the present study, among which PPOP, the one with relatively flexible backbone, showed superior transfection efficiency and cytotoxicity than the other two analogs, PPTP, and PPIP (Figures 4, 5, and 8). The higher flexibility of the polymer backbone resulted from larger cross-linking loops in PPOP facilitated the better accessibility of its cationic groups to reach the anionic sites of nucleic acids and cell membranes. For a practical nucleic acid delivering cationic polymer, however, the parallel association between transfection efficiency and cytotoxicity should best be reversed. Such high-transfection efficiency but low toxicity nature was achieved by one of our recently reported polyimines, PSI (Duan et al., 2012). In PSI, the aromatically conjugated bisimine linkage involved an imidazole ring for which the $\mathrm{pKa}$ is 5.9, slightly higher than the endosome $\mathrm{pH}$. For PPOP, this non-toxicity nature may easily be achieved by replacing the OP with the same cis-positioned imidazole-4,5-formaldehyde in the polymerization reaction. Replacement by imidazole-4,5formaldehyde may also resolve another reported cytotoxicity source from OP simultaneously (Morinaga et al., 2010; Iwasawa et al., 2011). As discussed above, such internally cross-linked yet still sufficiently flexible network of PPOP may be an ideal cationic polymer for gene delivery for its enhanced overall backbone stability and nontoxic degrading products (PEI-1800 and imidazole-4,5-formate; Duan et al., 2012).

\section{CONCLUSION}

Cationic polyimines polymerized through aromatically conjugated bis-imine linkages of various substitution positions is a new class of nucleic acid packing polymers for intracellular

\section{REFERENCES}

Akinc, A., Lynn, D. M., Anderson, D. G., and Langer, R. (2003). Parallel synthesis and biophysical characterization of a degradable polymer library for gene delivery. J. Am. Chem. Soc. 125, 5316-5323. doi: 10.1021/ja0 $34429 c$

Convertine, A., Diab, C., Prieve, M., Paschal, A., Hoffman, A., Johnson, P., et al. (2010). pH-responsive polymeric micelle carriers for siRNA drugs. Biomacromolecules 11, 2904-2911. doi: 10.1021/bm100652w

Duan, S., Yuan, W., Wu, F., and Jin, T. (2012). Polyspermine imidazole-4, 5imine, a chemically dynamic and biologically responsive carrier system for intracellular delivery of siRNA. Angew. Chem. Int. Ed. Engl. 51, 7938-7941. doi: 10.1002/anie. 201201793

Ge, X., Duan, S., Wu, F., Feng, J., Zhu, H., and Jin, T. (2015). Polywraplex, functionalized polyplexes by post-polyplexing assembly of a rationally designed triblock copolymer membrane. Adv. Funct. Mater. 25, 4352-4363. doi: 10.1002/adfm.201500724

Ginn, S. L., Alexander, I. E., Edelstein, M. L., Abedi, M. R., and Wixon, J. (2013). Gene therapy clinical trials worldwide to 2012-an update. J. Gene Med. 15, 65-77. doi: 10.1002/jgm.2698

Hong, S., Leroueil, P. R., Janus, E. K., Peters, J. L., Kober, M.-M., Islam, M. T., et al. (2006). Interaction of polycationic polymers with supported lipid bilayers and cells: nanoscale hole formation and enhanced membrane permeability. Bioconjug. Chem. 17, 728-734. doi: 10.1021/bc06 $0077 y$ delivery characterized with designable $\mathrm{pH}$-responsiveness, stability and backbone flexibility. When a highly branched low MW starter bearing multiple amino groups is allowed to polymerize with aromatic bis-aldehydes, the ortho-substituted aldehyde linker may only form conjugated bis-imine linkages of trans-configuration due to steric hindrance. This steric restriction may result in internally cross-linked polymers of relatively larger cross-linking loops as compared with those formed from tera- or iso-substituted aromatic bis-aldehydes. The relatively larger cross-linking loops endow the formed cationic polymer more flexible overall backbone that facilitates the electrostatic condensation of nucleic acids as well as the electrostatic adsorption onto cell membranes, leading to enhanced transfection efficiency and cytotoxicity. Using imidazole-4,5-dialdehyde, an ortho-substituted aromatic bisaldehyde involving a heterogeneous five-membered ring, to polymerize the highly branched starter should resolve the cytotoxicity and retain the transfection activity of the formed polymer.

\section{AUTHOR CONTRIBUTIONS}

SC designed the experiments with TJ, carried out the experiments, and drafted the manuscript. TJ initiated this study, involved the experiment design, provided continuous discussion, and finalized the manuscript.

\section{ACKNOWLEDGMENT}

The authors thank the Analytical Center of Shanghai Jiao Tong University for technical support.

Iwasawa, A., Niwano, Y., Kohno, M., and Ayaki, M. (2011). Bactericidal effects and cytotoxicity of new aromatic dialdehyde disinfectants (ortho-phthalaldehyde). Biocontrol Sci. 16, 165-170. doi: 10.4265/bio.16.165

Kanasty, R., Dorkin, J. R., Vegas, A., and Anderson, D. (2013). Delivery materials for siRNA therapeutics. Nat. Mater. 12, 967-977. doi: 10.1038/nma $\mathrm{t} 3765$

Lafourcade, C., Sobo, K., Kieffer-Jaquinod, S., Garin, J., and Van Der Goot, F. G. (2008). Regulation of the V-ATPase along the endocytic pathway occurs through reversible subunit association and membrane localization. PLoS ONE 3:e2758. doi: 10.1371/journal.pone.0002758

Lee, Y. S., and Kim, S. W. (2014). Bioreducible polymers for therapeutic gene delivery. J. Control. Release 28, 424-439. doi: 10.1016/j.jconrel.2014. 04.012

Lee, Y. S., Pressman, S., Andress, A. P., Kim, K., White, J. L., Cassidy, J. J., et al. (2009). Silencing by small RNAs is linked to endosomal trafficking. Nat. Cell Biol. 11, 1150-1156. doi: 10.1038/ncb1930

Lukacs, G. L., Haggie, P., Seksek, O., Lechardeur, D., Freedman, N., and Verkman, A. (2000). Size-dependent DNA mobility in cytoplasm and nucleus. J. Biol. Chem. 275, 1625-1629. doi: 10.1074/jbc.275.3. 1625

Lv, H., Zhang, S., Wang, B., Cui, S., and Yan, J. (2006). Toxicity of cationic lipids and cationic polymers in gene delivery. J. Control. Release 114, 100-109. doi: 10.1016/j.jconrel.2006.04.014

Mintzer, M. A., and Simanek, E. E. (2009). Nonviral vectors for gene delivery. Chem. Rev. 109, 259-302. doi: 10.1021/cr800409e 
Morinaga, T., Hasegawa, G., Koyama, S., Ishihara, Y., and Nishikawa, T. (2010). Acute inflammation and immunoresponses induced by ortho-phthalaldehyde in mice. Arch. Toxicol. 84, 397-404. doi: 10.1007/s00204-010-0512-1

Nguyen, J., and Szoka, F. C. (2012). Nucleic acid delivery: the missing pieces of the puzzle? Acc. Chem. Res. 45, 1153-1162. doi: 10.1021/ar3000162

Overly, C. C., Lee, K.-D., Berthiaume, E., and Hollenbeck, P. J. (1995). Quantitative measurement of intraorganelle $\mathrm{pH}$ in the endosomal-lysosomal pathway in neurons by using ratiometric imaging with pyranine. Proc. Natl. Acad. Sci. U.S.A. 92, 3156-3160. doi: 10.1073/pnas.92.8.3156

Pack, D. W., Hoffman, A. S., Pun, S., and Stayton, P. S. (2005). Design and development of polymers for gene delivery. Nat. Rev. Drug Discov. 4, 581-593. doi: $10.1038 / \mathrm{nrd} 1775$

Plank, C., Oberhauser, B., Mechtler, K., Koch, C., and Wagner, E. (1994). The influence of endosome-disruptive peptides on gene transfer using synthetic virus-like gene transfer systems. J. Biol. Chem. 269, $12918-12924$.

Rekha, M., and Sharma, C. P. (2009). Blood compatibility and in vitro transfection studies on cationically modified pullulan for liver cell targeted gene delivery. Biomaterials 30, 6655-6664. doi: 10.1016/j.biomaterials.2009. 08.029
Stalder, L., Heusermann, W., Sokol, L., Trojer, D., Wirz, J., Hean, J., et al. (2013). The rough endoplasmatic reticulum is a central nucleation site of siRNA-mediated RNA silencing. EMBO J. 32, 1115-1127. doi: 10.1038/emboj. 2013.52

Stenmark, H. (2009). Rab GTPases as coordinators of vesicle traffic. Nat. Rev. Mol. Cell Biol. 10, 513-525. doi: 10.1038/nrm2728

Yin, H., Kanasty, R. L., Eltoukhy, A. A., Vegas, A. J., Dorkin, J. R., and Anderson, D. G. (2014). Non-viral vectors for gene-based therapy. Nat. Rev. Genet. 15, 541-555. doi: $10.1038 / \mathrm{nrg} 3763$

Conflict of Interest Statement: The authors declare that the research was conducted in the absence of any commercial or financial relationships that could be construed as a potential conflict of interest.

Copyright (C) 2016 Chen and Jin. This is an open-access article distributed under the terms of the Creative Commons Attribution License (CC BY). The use, distribution or reproduction in other forums is permitted, provided the original author(s) or licensor are credited and that the original publication in this journal is cited, in accordance with accepted academic practice. No use, distribution or reproduction is permitted which does not comply with these terms. 